\title{
Improvement of Human-Machine Interaction with Applications to Information Retrieval System
}

\author{
Vitaliy Vitsentiy
}

\begin{abstract}
A human-machine system, where human forms a control target for an automatic control system and estimates feedback is considered. An adaptive control system (ACS) which on each control step, except the criterion of control optimality, has the criterion to provide more information from human is offered. The system adapts to combine them for achievement of optimality of several controls. As an example of the ACS an information retrieval system (IRS) is offered.
\end{abstract}

Index Terms-Adaptive control, human-machine interaction, information-retrieval system.

\section{INTRODUCTION}

Though development of the systems, which use dialogue with humans, began from the start of computer usage [1], nowadays there are not systems, capable to perceive the natural language perfectly. Formalized languages are used for interaction with human [2]. Generally, translation from the natural language into a formalized is inconvenient, leads to errors and inaccuracy. Among the reasons is that user's information can be fuzzy, because of fuzziness of the natural language and the feature of humans to use intuitive fuzzy patterns.

Human-machine interaction (HMI) can be improved with the help of the following adaptive control system (ACS). The system performs each control task several times. On each control step the system tries not only to ensure control optimality but also to increase information from the user. Such control is dual [3]. In traditional dual control systems it is necessary to identify control object or explore the disturbances, which act on object, which is different from the increase of information quantity obtained from human. Information quantity increase is in feedback and more precise control target. Providing of the striving for obtaining more information can lead to smaller optimality of some separate control but to greater optimality of several controls.

\section{AdAPTIVE CONTROL}

Let's consider a traditional approach to adaptive

V.Vitsentiy is with the Institute of Computer Information Technologies, Ternopil Academy of National Economy, Ternopil, Ukraine (telephone: +380 (352) 330830, e-mail: vv@tanet.edu.te.ua). control represented in the formulas (1) - (5). State vector of control object has the following functional expression:

$$
x_{k+1}=\phi_{k}\left(x_{x}, u_{k}, w_{k}\right)
$$

where $x_{k}$ - state vector of control object on $k$ control step; $\phi_{k}-$ some function; $u_{k}$ - control vector; $w_{k}$ - vector of disturbances.

The task of adaptive control consists in finding the optimal control, which transforms the system into the state, which is the most close to $t_{k+1}$. Control vector has the following functional expression:

$$
u_{k}=\gamma_{k}\left(\theta_{k}, x_{k}, t_{k+1}, w_{k}\right)
$$

where $\gamma_{k}$ - control law; $\theta_{k}$ - parameters of control law; $t_{k+1}-$ control target.

This task is equivalent to evaluation of parameters $\theta$, which minimize some optimality criterion of control $J_{k}\left(\theta_{k}\right)$, which can be for example the following:

$$
J_{k}\left(\theta_{k}\right)=E\left(\left(x_{k+1}-t_{k+1}\right)^{T} A_{k}\left(x_{k+1}-t_{k+1}\right)+u_{k}{ }^{T} B_{k} u_{k}\right)
$$

where $A_{k}, B_{k}$ - wage matrixes.

Vector $\theta_{k}$ can be found using gradient $g_{k}\left(\theta_{k}\right)$ :

$$
g_{k}\left(\theta_{k}\right)=\frac{\partial J_{k}}{\partial \theta_{k}}=\frac{\partial u_{k}^{T}}{\partial \theta_{k}} \frac{\partial J_{k}}{\partial u_{k}}=0
$$

in:

$$
\hat{\theta}_{k}=\hat{\theta}_{k-1}-\alpha_{k} g_{k}\left(\theta_{k}\right)
$$

where $\alpha_{k}-$ scalar, which defines a search speed.

$$
\text { It is complicated to analytically define } \frac{\partial J_{k}}{\partial u_{k}} \text { and } \frac{\partial J_{k}}{\partial \theta_{k}}
$$

for some stochastic systems. In such case for gradient estimation it is possible to use methods of approximation on the basis of $J$ values. For this purpose Simultaneous 
Perturbation Stochastic Approximation (SPSA) algorithm is appropriate [4], [5]. According to it the estimation of $\hat{g}_{k}\left(\theta_{k}\right)$ is equal:

$$
\hat{g_{k}}\left(\hat{\theta_{k}}\right)=\frac{J\left(\hat{\theta}_{k}+c_{k} \Delta_{k}\right)-J\left(\hat{\left.\theta_{k}-c_{k} \Delta_{k}\right)}\right.}{2 c_{k}}\left[\begin{array}{l}
\Delta_{k 1}^{-1} \\
\Delta_{k 2}{ }^{-1} \\
\cdots \\
\cdots . . \\
\Delta_{k p}^{-1}
\end{array}\right](6
$$

where $\Delta_{k i}-$ random variable distributed according to Bernoulli distribution, which assigns values $\pm 1 ; c_{k}$ - vector of absolute values of parameters perturbations.

The numerator in expression (6) has one value for all components of vector $\theta_{k}$. Insignificant number of measurements of optimality criterion $J$ for evaluation of $\hat{g}_{k}\left(\theta_{k}\right)$ makes the basic advantage of the method above other methods of adaptive control, for example above finite-difference gradient approximation [6] or a method of random search.

\section{AN ACS WITH PROVIDING MoRE INFORMATIVE HMI}

In fig. 1 an example of a control system with HMI is depicted. Human performs system functional parts, enclosed by the dashed line, machine - others. HMI depicted on fig. 1, 2 and 3 is constructed in such a manner that human and machine make a control system of the higher level. The machine functional parts are the control object for human but they can be considered as a separate control system of lower level.

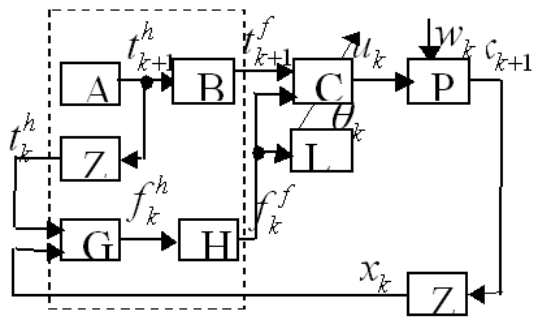

Fig. 1. Control system with HMI.

where A - forming of control target; B - formalization of control target; $\mathrm{C}$ - controller of the control system of the lower level; $\mathrm{P}$ - control object; G - comparison of control target and control object state; H formalization of the comparison result; L - learning mechanism; Z delay; $t_{k+1}^{h}$ - control target on $k$ 'th control step; $t_{k+1}^{f}$ - formalized control target; $f_{k}^{h}$ - value of comparison of control object state $x_{k}$ and control target $t_{k}^{h} ; f_{k}^{f}$ - formalized value of control target $f_{k}^{h}$.

Sometimes a person organizes performing of a control task several times and uses even an ACS as a control object (fig. 2 and 3). The person uses such series of repeated controls in particular for better comprehension of the task and control target, for study of the control object, disturbances, which act on it, and accordingly for improvement of the control target and providing the system with an better evaluation of the feedback. The person takes into consideration intuition, knowledge, possibilities of information perception etc. Machine performs rather specialized function with adaptation. The adaptation loops of both control systems are of higher and lower level.

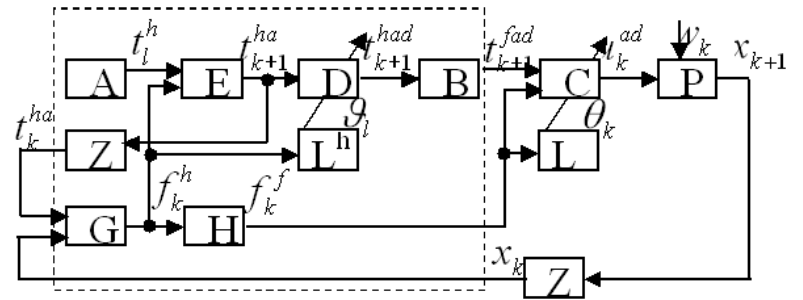

Fig. 2. Control system where human uses control series.

where E - more precise definition of control target; D - correction of control target to provide dual criteria; $\mathrm{L}^{\mathrm{h}}$ - human's learning mechanism; $t_{l}^{h}$ - control target of 1 'th series of controls; $t_{k+1}^{h a}-$ control target of $k+1$ repeated control; $t_{k+1}^{\text {had }}-$ corrected control target $t_{k+1}^{h a}$ to provide dual criteria; $t_{k+1}^{f a d}$ - formalized control target $t_{k+1}^{h a d}$; $u_{k}^{a d}$ - control vector; $\vartheta_{l}$ - parameters of control law, which correspond to the adaptation on higher level.

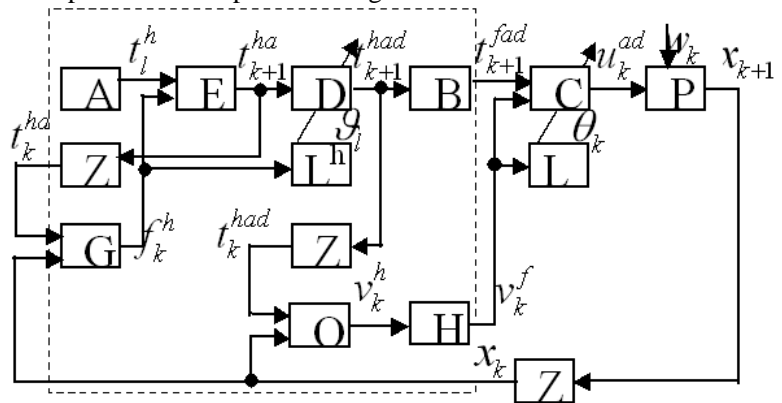

Fig. 3. Control system with two comparisons of feedback.

where Q - comparison of control target and state of control object for the system; $v_{k}^{h}$ - value of comparison of control target and state of control object, calculated for the system; $v_{k}^{f}$ - formalized value of comparison $v_{k}^{h}$.

Among the drawbacks of the control systems, depicted in fig. 2 and 3, there are the following: 1) human is entrusted with providing of duality; 2) human is entrusted with adaptation to provide duality control targets; 3) human differently evaluates feedback for the machine system and for himself.

The offered control system eliminates these drawbacks (fig. 4). In this system machine are entrusted with the function to provide duality and adaptation.

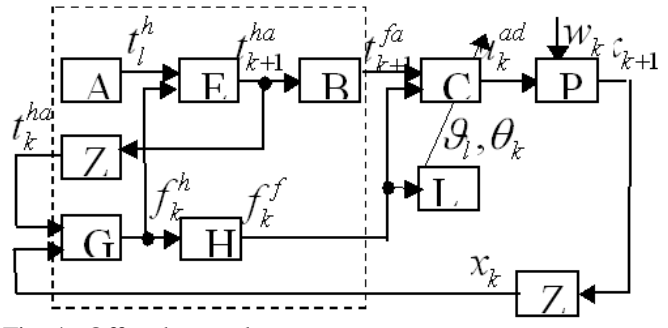

Fig. 4. Offered control system.

Parameters of control law divide into corresponded to 
the adaptation of higher and lower level. Control law now is:

$$
u_{k}=\gamma_{k}\left(\vartheta_{l}, \theta_{k}, x_{k}, t_{k+1}, w_{k}\right)
$$

Parameters $\vartheta_{l}$ adjust after each series of repeated controls $\left\{u_{k}\right\}, k \in L_{l}$ where $L_{l}$ is $l$ series of repeated controls. Because more optimality of a control series can correspond with nonoptimality of some controls in the series. At that a control optimality criterion of control series serves as the two criteria of the lower level. A control algorithm with two levels adaptation is depicted in fig. 5. Optimality criterion (3) can be transformed:

$$
J_{l}\left(\vartheta_{l}, \theta_{k}\right)=E \sum_{k \in L_{l}}\left(x_{x}-t_{k}\right)^{T} A_{k}\left(x_{k}-t_{k}\right)+u_{k}{ }^{T} B_{k} u_{k}
$$

Forming of more informative HMI in the offered system is obtained by the principle: the more information a person has as the result of a control, the more information the ACS obtains from the person at feedback evaluation and defining more precise the control target. The machine part of the system should form such controls each of them is both optimal on its control step and informative. The system should find the optimal join of these criteria at adaptation.

For realization of adaptation it is difficult to apply the methods, which are based on gradient of control optimality criterion. Because: 1) human at least compares control target and feedback; 2) it is necessary to derive gradient for optimality criterion of series of repeated controls. Instead of the methods, which use analytical expression of gradient, it is possible to use the methods of gradient approximation. The advantage of SPSA over them is based on the amount of optimality criterion measurements. Emphasize the following peculiarities for the offered system: 1) for measurement of one value of criterion it is necessary to carry out a series of controls; 2) different criterion measurements correspond with different control targets. Thus, each next value of optimality criterion is measured when the system has considerably changed. In such case many measurements for evaluation of a value of gradient will result in inaccuracy. Among different forms of SPSA the onemeasurement is better in the systems with significant changes of dynamics [7].

\section{AN APPROACH to CONSTRUCTION OF IRS}

Information retrieval system (IRS) is a convenient example for realization of the above theoretical aspects. While working with an IRS a user creates a query for information search. The IRS should give the required information. Usually IRS does not give the information at once, it does this in portions due to the reasons that a user can not apprehend a lot of information at once and that there is a necessity to limit the time of the answer for a query. This feature of IRS can be used for construction of the system that provides more informative interaction with human without changing anything in the instructions of IRS usage.

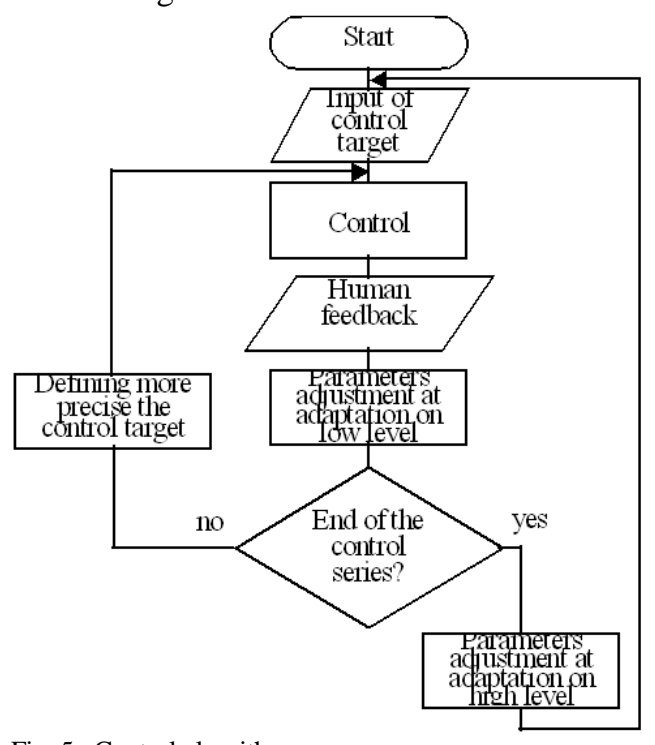

Fig. 5. Control algorithm.

Relatively to IRS the adaptation on the lower level concerns correct definition of the correspondence between every document and a query. The adaptation on the higher level concerns evaluation whether a document gives more information, if it is included in the portion.

The control law of IRS defines relevance of documents, i.e. the values, which represent the correspondence between documents and a query and are used to define the order of presenting the documents for the user. Take the following optimality criterion

$$
J_{l}\left(\vartheta_{l}\right)=\sum_{p} \sum_{k}\left(x_{p k l}-t_{p k l}\right)^{2}
$$

where $\vartheta_{l}$ - vector of parameters of control law for l'th query; $x_{p k l}-$ relevance value of $k^{\prime}$ th document in $p^{\prime}$ th documents portion to $l^{\prime}$ th query; $t_{p k l}$ - relevance value defined by user, let's assume, it is known.

While answering a query, relevance is defined for the documents, which have not been given to the user yet and the documents with the greatest relevance are included in the current portion.

\section{OFFERED IRS}

The schema of the IRS control system is depicted in fig. 6. The task it performs is similar to pattern recognition. The IRS provides increase of information in evaluation of feedback by a user.

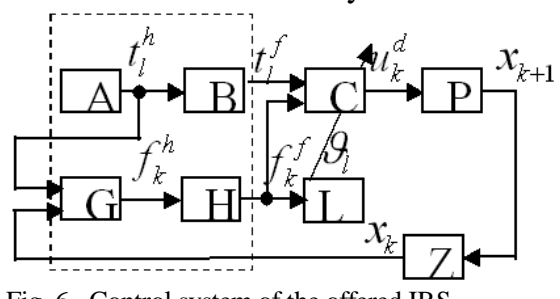

Fig. 6. Control system of the offered IRS

Let's take a neural network as an approximation of the 
control law. The known methods of neural network learning on the basis of back propagation algorithm can not be used for the offered IRS because this algorithm requires analytical definition of optimality criterion gradient. For neural network learning the methods on the basis of SPSA [8], [9] can be used.

The neural network output value defines relevance of a document. The neural network has the following input data: document pattern, query pattern, feedback of relevance increase pattern, feedback of relevance decrease pattern, pattern of the documents which have been included in the current portion already. These patterns are formed by appropriate algorithms and look like: $\left(x_{1}, x_{2}, \ldots, x_{i}, \ldots, x_{m}\right)^{T}$. Here $x_{i}$ is the value of presence of $i$ 'th word in a pattern, it assigns a value from 0 up to 1 .

For this neural network the mechanism of providing more informative interaction can be described as the following. Feedback patterns are generated on the basis of the documents given to the user in a portion of documents. Let's assume that the portion with more different documents has more information. For forming such portions the neural network during evaluation of the relevance of a document not only takes into account the correspondence between the document, query and feedback patterns but also the difference between the pattern of the documents, which have been included already in the portion and the pattern of the current document.

\section{EMPIRICAL STUDIES}

In this section is considered an example of work of the offered IRS, which besides the criterion to correctly define documents relevance has the criterion to provide more information from humans and a usual IRS, which does not have this additional criterion.

Consider the query to IRS: "natural energy resources". In the following tables 1 and 2 the answers of the systems are written out. For each document the following relevance values to the query are indicated: 1) estimated by system $-x ; 2$ ) determined by the user $-t ; 3$ ) estimated by the system without use of feedback $-x^{\prime} . x^{\prime}$ is necessary for revealing the modifications, which are introduced by feedback and by providing more informative $\mathrm{CHI}$. The last column of the tables comprises values of $J$, which are calculated according to (9) for each document, portion of documents, three portions of documents.

One portion comprises five documents. The words, which relate the documents to the query, are marked by bold type. It is possible to divide documents in the database of the IRS into groups with common subjects. The words, which identify documents with a group are marked by italic type, if one of them is included in a feedback pattern then it influences estimation of relevance of the remaining documents. These words are written in the tables after each portion: with a mark "+" which increase relevance, with a mark "-" which decrease relevance. In the following portions these words influence relevance and are marked by bold type.

Comparing tables 1 and 2 it is seen that the offered IRS provides more words of feedback. Thus, the system retrieves more information from the user.

First portions of the documents in both tables have different fifth document. Fifth document of table 1 is included in the first portion according to its estimated relevance $x^{\prime}=x$. Fifth document of table 2 has $x^{\prime}$ equal to 0.85 and is included in first portion of table 2 , because it differs from the other documents of the portion and consequently its relevance $x$ was increased up to 0.9 . Fifth document of table 1 is similar to second document. Therefore, in table 2 while estimating its relevance it was less than or equal to $x^{\prime}$, that has not permitted the document to be included in the portion. The inclusion of fifth document in first portion of table 2 provided an additional feedback word "sun". This word is considered as such, that increases relevance in table 2 starting with second portion of documents and is marked by bold type. In table 1 this word influences relevance estimation starting with forth portion.

In second portion in table 1 there are three similar documents, which have a common word "energetic", but have small user's relevance. In table 1 there is only one document with this word because relevance of the other documents was decreased due to their similarity.

More informative interaction with user of the offered IRS for described relatively small examples in tables 1 and 2 has provided more optimal value of optimality criterion in 1.35 times than another IRS.

\section{CONCLUSIONS}

The offered ACS for human-machine systems forms dual control. The criteria it has are the following: to provide optimal control on a control step and to retrieve more information from a person. The system adapts to combine them for achievement of optimality of control series.

As an example of realization of the approach an IRS is offered in the paper. There were considered examples of human interaction with the offered and usual IRS. The optimality criterion of the former was 1.35 times better. Other applications can be a control system of the robot, which performs human commands; a system of adjustment of human-computer interface; a system of conversation with a person; a control system in multiagent environment; a system of multilevel pattern recognition.

Directions of further researches are development of applied systems, methods of multilevel adaptation, methods of neural network learning and development of their architecture for multilevel adaptation, principles of information increase in human-machine interaction and their concrete definition for applications and other. 
TABLE I

THE ANSWERS OF THE USUAL IRS

\begin{tabular}{|c|c|c|c|c|}
\hline \multirow[t]{2}{*}{ Documents } & \multicolumn{3}{|c|}{ Relevance } & \multirow{2}{*}{$J$} \\
\hline & $X^{\prime}$ & $x$ & $t$ & \\
\hline 1. A program of use of natural energy resources. & 0.9 & 0.9 & 0.9 & 0 \\
\hline 2. Importance of use of natural energy resources for Ukrainian economy. & 0.9 & 0.9 & 0.9 & 0 \\
\hline $\begin{array}{l}\text { 3. The cheap price of natural energy resources is not the basic reason of their use, solution } \\
\text { of ecological problems is also very important. }\end{array}$ & 0.9 & 0.9 & 0.9 & 0 \\
\hline $\begin{array}{l}\text { 4. The atomic energy is an important energy resource and provides only advantages if not } \\
\text { to consider harm, which it puts to a nature. }\end{array}$ & 0.9 & 0.9 & 0.2 & 0.49 \\
\hline 5. Ukrainian natural energy resources. A program of use. & 0.9 & 0.9 & 0.2 & 0.49 \\
\hline \multicolumn{4}{|l|}{$\begin{array}{l}\text { + ecological } \\
\text { - atomic }\end{array}$} & 0.98 \\
\hline $\begin{array}{l}\text { 6. It is necessary to find the balance in use of natural energy of gas for the economy and } \\
\text { losses of nature. }\end{array}$ & 0.85 & 0.85 & 0.8 & 0.0025 \\
\hline 7. The map of resources of natural energy of gas of Ukraine. & 0.85 & 0.85 & 0.8 & 0.0025 \\
\hline 8. Energetic use of resources of a nature. & 0.85 & 0.85 & 0.1 & 0.5625 \\
\hline 9. Usage of natural resources is energetic and this does not help ecology. & 0.85 & 0.85 & 0.1 & 0.5625 \\
\hline $\begin{array}{l}\text { 10. It is necessary to stop the energetic felling of woods for restoration of natural resources } \\
\text { of a wood. }\end{array}$ & 0.85 & 0.85 & 0 & 0.7225 \\
\hline \multicolumn{5}{|l|}{ - energetic, gas } \\
\hline 11. Large cheap natural resources of the sun energy. & 0.85 & 0.85 & 0.85 & 0.0025 \\
\hline $\begin{array}{l}\text { 12. An approach to use of energy of the sun, wind, water and gas as cheap, ecological } \\
\text { energy resources. }\end{array}$ & 0.75 & 0.75 & 0.85 & 0.01 \\
\hline $\begin{array}{l}\text { 13. Natural resources of human mental energy. The report about consequences of use } \\
\text { energizers, drugs, mental preparations. }\end{array}$ & 0.8 & 0.8 & 0 & 0.64 \\
\hline 14. Psychological processes during restoration of human natural mental energy resources. & 0.7 & 0.8 & 0 & 0.64 \\
\hline $\begin{array}{l}\text { 15. The negotiation between Ukraine and Hungary about an opportunity of common use of } \\
\text { natural energy has passed. }\end{array}$ & 0.7 & 0.7 & 0.8 & 0.01 \\
\hline $\begin{array}{l}\text { + sun, water } \\
- \text { mental }\end{array}$ & & & & 1.3. \\
\hline
\end{tabular}

TABLE II

THE ANSWERS OF THE OFFERED IRS

\begin{tabular}{|c|c|c|c|c|}
\hline \multirow[t]{2}{*}{ Documents } & \multicolumn{2}{|c|}{ Relevance } & \multirow[b]{2}{*}{$t$} & \multirow[b]{2}{*}{$J$} \\
\hline & & $x$ & & \\
\hline A program of use of natural energy resources. & 0.9 & 0.9 & 0.9 & 0 \\
\hline Importance of use of natural energy resources for Ukrainian economy. & 0.9 & 0.9 & 0.9 & 0 \\
\hline $\begin{array}{l}\text { 3. The cheap price of natural energy resources is not the basic reason of their use, solution } \\
\text { of ecological problems is also very important. }\end{array}$ & 0.9 & 0.9 & 0.9 & 0 \\
\hline $\begin{array}{l}\text { 4. The atomic energy is an important energy resource and provides only advantages if not } \\
\text { to consider harm, which it puts to a nature. }\end{array}$ & 0.9 & 0.9 & 0.2 & 0.49 \\
\hline 5. Large cheap natural resources of the sun energy. & 0.85 & 0.9 & 0.9 & 0 \\
\hline $\begin{array}{l}\text { + sun, ecological } \\
\text { - atomic }\end{array}$ & & & & 0.49 \\
\hline 6. Ukrainian natural energy resources. A program of use. & 0.9 & 0.9 & 0,9 & 0 \\
\hline $\begin{array}{l}\text { 7. It is necessary to find the balance in use of natural energy of gas for the economy and } \\
\text { losses of nature. }\end{array}$ & 0.85 & 0.85 & 0.8 & 0.0025 \\
\hline 8. $\quad$ Energetic use of resources of nature. A program of use. & 0.85 & 0.85 & 0.1 & 0.5625 \\
\hline 9. The farmers are very energetic in allotment of natural ground resources. & 0.85 & 0.85 & 0 & 0.7225 \\
\hline 10. The natural wind and sun energy is the cheapest source of energy. & 0.75 & 0.8 & 0.8 & 0 \\
\hline $\begin{array}{l}\text { wind } \\
\text { - ground, energetic, gas }\end{array}$ & & & & 1.2875 \\
\hline $\begin{array}{l}\text { 11. The approach to the use of energy of sun, water, and gas as cheap, ecological energy } \\
\text { resources. }\end{array}$ & 0.75 & 0.85 & 0.85 & 0 \\
\hline 12. The map of resources of natural energy of gas of Ukraine. & 0.85 & 0.8 & 0.8 & 0 \\
\hline 13. The researches into ecological safety of energy resources. & 0.7 & 0.8 & 0.8 & 0 \\
\hline $\begin{array}{l}\text { 14. Natural resources of human mental energy. The report about consequences of use } \\
\text { energizers, drugs, mental preparations. }\end{array}$ & 0.8 & 0.8 & 0 & 0.64 \\
\hline 15. Resources of spiritual energy of nature & 0.8 & 0.8 & 0 & 0.64 \\
\hline $\begin{array}{l}\text { + safe, water } \\
\text { - mental, spiritual }\end{array}$ & & & & 1.28 \\
\hline
\end{tabular}




\section{REFERENCES}

[1] V. Drakin, E. Popov, A. Preobrazhensky intercourse of end users with data processing systems. Radio e svyas, Moscow, 1988. (in Russian).

[2] E. Popov Intercourse with computer in natural language. Nauka, Moscow, 1982. (in Russian).

[3] A. A. Feldbaum Fundamentals of the theory of optimal automatic systems. Nauka, Moscow, 1966. (in Russian).

[4] J. C. Spall Implementation of the simultaneous perturbation algorithm for stochastic optimization. IEEE Transactions on Aerospace and Electronic Systems, 34, 1998, pp. 817-823.

[5] J. C. Spall Multivariate stochastic approximation using a simultaneous perturbation gradient approximation. IEEE Transactions on Automatic Control, 37, 1992, pp. 332-341.

[6] J. Kiefer, J. Wolfowitz Stochastic estimation of a regression function. Annals of Mathematical Statistics, 23, 1952, pp. 462-466

[7] J. C. Spall A one measurement form of simultaneous perturbation stochastic approximation, Automatica 33 (1997), pp.109-112.

[8] A. Vande Wouwer, C. Renotte, M. Remy On the use of simultaneous Perturbation stochastic approximation for neural network training, Proceedings of the American Control Conference, San Diego, California, June 1999, pp. 388-392.

[9] Y. Maeda, R. J. P. De Figueiredo, Learning rules for neurocontroller via simultaneous perturbation, IEEE Transactions on Neural Networks, 8 (5), September 1997, pp. 1119-1130. 